\title{
Monitoring drinking: A method compatible with the recording of electrophysiological phenomena'
}

E U G E N E E I S M A N , UN I VER S I TY
WISCONSIN-MILWAUKEE, Milwaukee, Wisconsin 53201

The Hill-Stellar method of monitoring drinking behavior has proven effective in many applications. Because of the introduction of electrical artifacts, however, it is incompatible with certain procedures for recording electrophysiological phenomena. An alternative method is described that avoids such artifacts but still permits the lick-by-lick monitoring of drinking behavior.

Hill and Stellar (1951) report a method for monitoring drinking behavior in which a current-sensitive relay (drinkometer) is used to detect each lap of the tongue as an animal drinks from a typical laboratory water tube. Although an electric current passes through the animal during each lap of the tube, the magnitude is so small that behavioral effects due to its passage are presumably absent. The method has proven effective, therefore,

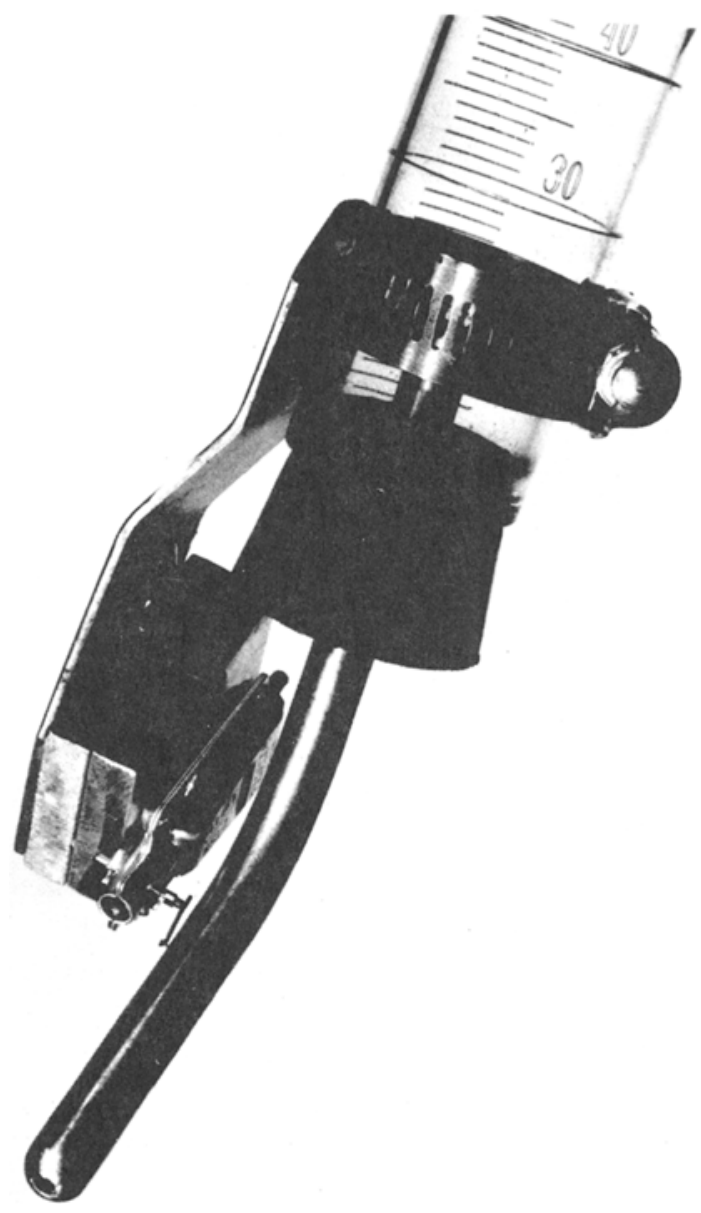

Fig. 1. Bracket and cartridge assembly, mounted on a water cylinder, with the drinking tube in place.

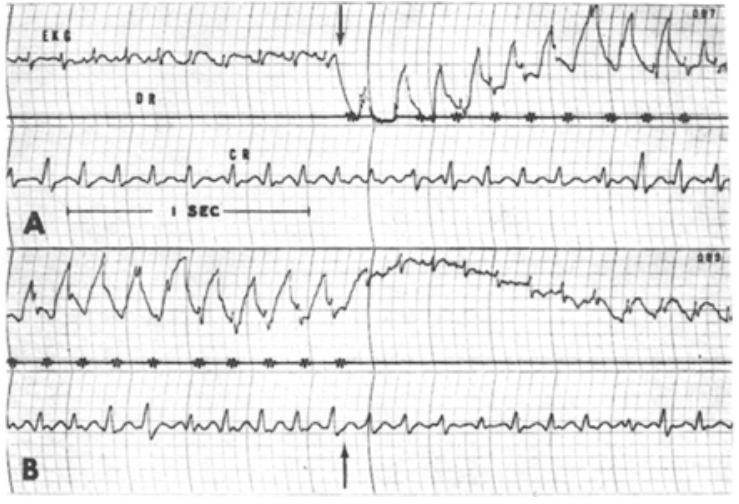

Fig. 2. A polygraph record showing the presence or absence of artifacts in the electrocardiogram (EKG) when drinking is monitored with a drinkometer (DR) or a crystal (CR), respectively. The drinkometer was turned on at the downward arrow and off at the arrow pointing up.

in most applications. When the attempt is made to monitor drinking in this manner, and to record certain electrophysiological phenomena at the same time, however, even that low a current is sufficient to produce intolerable artifacts in the record. An alternative method is presented in this paper that eliminates these artifacts but still permits drinking to be monitored in a lick-by-lick fashion.

In the new method, a simple crystal phonograph cartridge (Astatic Co., No. 62-1) is mounted with its stylus in contact with the drinking tube, as shown in Fig. 1. When the animal licks the tube, movement of the stylus occurs and a characteristic voltage is generated in the crystal. This voltage can then be amplified and recorded directly as an analog of the licking response, or it can be detected with a voltage-sensitive relay to provide a digital record of the lick-by-lick behavior as in the original method of Hill and Stellar (1951).

The polygraph records shown in Fig. 2 were obtained during a period in which a thirsty rat was permitted to drink continuously from a water tube fitted with a phonograph cartridge, in the manner described above. Provision was also made for monitoring the drinking behavior using the Hill-Stellar method, by connecting the floor of the cage and the drinking tube to the input of a commercially available drinkometer (Grason-Stadler Co.). The output of the drinkometer was used to operate a pulse former that provided a $50-\mathrm{msec}$ pulse to operate the signal marker on the polygraph. The output of the crystal was fed directly to one of the recording channels of the polygraph. ${ }^{2}$ Thus, in Strips $A$ and B of Fig. 1, the second and third tracings, from top to bottom, represent the records produced by the drinkometer and the crystal, respectively. The topmost tracing of each strip represents the electrocardiogram (EKG) of the rat, obtained according to procedures described elsewhere (Eisman, 1965). Paper speed for the records shown in Fig. 1 was $100 \mathrm{~mm} / \mathrm{sec}$. The animal had been drinking for a short time before, and during, the entire period represented in the recording. Strip B of the 
polygraph record is merely a continuation of Strip A, after 3 sec.

During the first portion of Strip A, the drinkometer had been turned off electrically. This is indicated by the absence of deflections in the second tracing. During this portion of the record, it can be seen that the EKG was stable and artifact-free and that licking responses were clearly indicated by the amplified output of the crystal. ${ }^{3}$ Midway through Strip A, the drinkometer was turned on. This event is indicated by the occurrence of deflections in the signal marker, which are perfectly correlated with the peaks in the record corresponding to the output of the crystal. At the same time, however, artifacts become apparent in the EKG tracing. The drinkometer remained on for approximately $6 \mathrm{sec}$, until a point midway through Strip B. When the drinkometer was turned off, the artifacts disappeared and within $1 \frac{1}{2} \mathrm{sec}$ the EKG was again readable and at the original base line. ${ }^{4}$ It is concluded, therefore, that the method for monitoring drinking behavior presented in this paper has all of the essential features of the original method (Hill \& Stellar, 1951) plus the important additional advantage of being compatible with procedures for recording electrophysiological phenomena.

\section{REFERENCES}

EISMAN, E. Technique for monitoring cardiac function without handling or retraining the animal. Perceptual \& Motor Skills, 1965, 20, 1093-1097.

HILL, J. H., \& STELLAR, E. An electronic drinkometer. Science, 1951, $114,43-44$.

\section{NOTES}

1. The work in this paper was supported by Grant No. 162-7442 from the Graduate School of the University of Wisconsin.

2. A Grass Model 5 polygraph, with a Model 5P1 preamplifier, was used to record the output of the crystal. The selector switch was set for a time constant of $.1 \mathrm{sec}$ and the high-frequency filter was set at 60 . The gain settings were such that a deflection of $1 \mathrm{~cm}=100 \mathrm{mV}$. The chart paper shown in Fig. 1 was printed with a spacing of $5 \mathrm{~mm}$ per box.

3. For digital measurements, a commercially available audio detection relay (Scientific Prototype Co.) has been used, with good success. The output of the crystal is fed directly to the input of the relay, with no preamplification required. Cumulative recorders and electromechanical counters can then be operated, as in the Hill-Stellar method.

4. The relatively long period of time required for restoration of the EKC trace is tentatively attributed to a property of the amplifier in the polygraph rather than to a persistent aftereffect, within the animal, of the passage of current from the drinkometer.

\section{Technique for monitoring activity in free-moving animals}

\author{
E U G E N E E I S M A N, UNIVERSITY \\ WISCONSIN-MIL WAUKEE, Milwaukee, Wisconsin 53201
}

$O F$

A techique is described for measuring activity by monitoring the rotational movements of a commutator, to which animals might be connected for other experimental purposes.

A method is presented in this paper for measuring behavioral activity in the rat. The technique is particularly appropriate for use with animals that would otherwise be prepared for electrophysiological recording or stimulation. In such preparations, overhead leads are usually carried from a terminal on the animal's head to a commutator situated above the experimental cage. It is the feature of the commutator that provides the means for measuring activity in this method.

A typical commutator is constructed of a circular disk with two concentric depressions to contain mercury. The disk rotates freely on a stationary vertical post through its center. Lead wires from the animal are connected to terminals on the underside of the disk, and through them, to the mercury contained in the depressions. A square cover is positioned above the disk, with the post through its center. Stationary electrodes project downward through the cover and into the mercury, to complete the circuit through the commutator. When animals are connected to the commutator, using properly counterweighted leads (Eisman, 1965), it is observed that even small movements are faithfully reflected in rotary movements of the disk. Activity can be measured by monitoring these rotary movements. The latter is accomplished by mounting a magnetic reed switch along one edge of the square cover and a small cylindrical magnet on the circumference of the disk. The long axes of the magnet, and the switch, are arranged parallel to the plane of rotation of the commutator. The switch is activated, therefore, each time the magnet passes beneath its center-point. Each closure of the switch can then be monitored on an electromagnetic counter, a

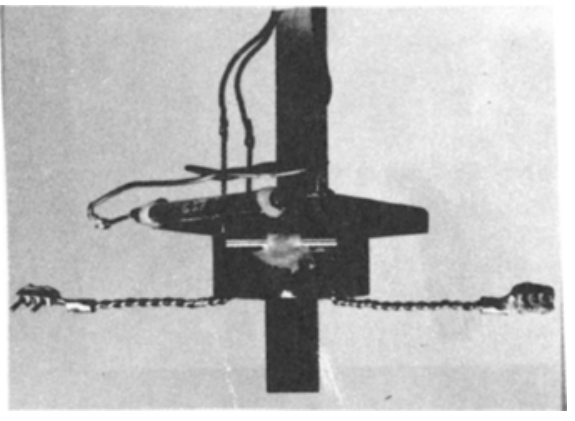

Fig. 1. A simple commutator with a reed switch mounted on the rotating disk and a magnet on the cover.

cumulative recorder, or the like, to obtain the measure of activity.

The sensitivity of the commutator system can be varied by using a configuration involving more than one reed switch. For example, it is possible to mount two switches, on opposite edges of the cover. Electrical circuitry can then be arranged so that a count is obtained from one of the switches, but only after the other switch has been closed at least once. Thus, if an animal were initially facing the front of the cage, it would have to turn toward the rear, and then in either direction back to the front, in order for a movement to be recorded. In this manner, it is possible to detect quite minor movements of the $\mathrm{S}$ or only those movements that yield some criterial degree of rotation in the commutator.

An application of the present method is illustrated in Fig. 2. A commutator with one reed switch, and a cumulative recorder were used to monitor the activity of a free-moving rat under two different temperature conditions. The animal was housed in an 